Diskussionspapiere des

Europäischen Instituts für Sozioökonomie e. V.

Working Papers of the

European Institute for Socioeconomics

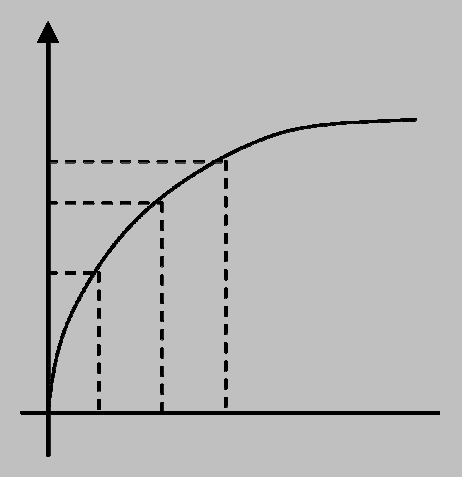

\title{
Unternehmer im Dopingmarkt - Gendoping als neues Geschäftsfeld
}

Eike Emrich \& Christian Pierdzioch

Nr. 13

Jahr 2015 


\section{Prof. Dr. Eike Emrich}

Fachbereich: Sportökonomie und Sportsoziologie

Adresse: $\quad$ Universität des Saarlandes

Sportwissenschaftliches Institut

Campus

66123 Saarbrücken

Tel: 049(0)6813024170

E-Mail: e.emrich@mx.uni-saarland.de

\section{Prof. Dr. Christian Pierdzioch}

Fachbereich: Makroökonomie und Internationale Wirtschaftsbeziehungen

Adresse: $\quad$ Helmut-Schmidt-Universität/Universität der Bundeswehr

Holstenhofweg 85

22043 Hamburg

Tel: 049(0)40 65412879

E-Mail: c.pierdzioch@hsu-hh.de

(c) 2015

Europäisches Institut für Sozioökonomie e. V. / European Institute for Socioeconomics

c/o Universität des Saarlandes

Sportwissenschaftliches Institut

Arbeitsbereich Sportökonomie und Sportsoziologie

Campus, Gebäude 8.1

66123 Saarbrücken

http://www.soziooekonomie.org 
Eike Emrich und Christian Pierdzioch

\section{Unternehmer im Dopingmarkt - Gendoping als neues Geschäftsfeld}

\section{$1 \quad$ Einleitung}

Die Entdeckungsquote identifizierter Dopingkonsumenten ist über die Zeit relativ stabil, wenn man die Aufklärungsquote von Welt-Anti-Doping-Agentur (WADA) bzw. Nationaler-AntiDoping-Agentur (NADA) in Deutschland betrachtet (vgl. Pitsch, Emrich \& Klein 2005, Pitsch, Maats \& Emrich 2009, Emrich \& Pitsch 2012, Pitsch 2009, 2011). Wie immer, wenn sich soziale Verhaltensweisen und institutionelle Regelungen trotz des Versuchs ihrer Eindämmung bzw. Bekämpfung als stabil und resistent gegenüber verschiedenen Änderungsversuchen erweisen (s. zur überindividuellen Klugheit institutioneller Regelungen Hayek 1969), sind aus einer (sozio-)ökonomischen Perspektive Gründe dafür zu vermuten, nämlich:

1. Es existieren status- und einkommensbezogene Interessen individueller und korporativer Akteure, die Einkommens- und Funktionsverluste fürchten und deshalb für den Erhalt ihres status quo und damit letztlich für den Erhalt materiellen und immateriellen Einkommens kämpfen.

2. Es existieren institutionelle Regelungen im Sport, die ein nachfragerelevantes Optimum zwischen Höchstleistung und Integrität der erbrachten Leistung ermöglichen und so Einkommenschancen stabilisieren.

3. Es ist zu vermuten, dass dieses Optimum auch angesichts exogener Schocks über die Zeit, getrieben durch die Interessen der Akteure, immer wieder in neue Gleichgewichtszustände mündet.

Im nachfolgenden Beitrag soll im Sinne einer ersten Skizze folgendes geleistet werden:

1) Wird der allgemeine soziale Konstruktionsmechanismus eines sozialen Problems auf das Phänomen Doping angewandt.

2) Wird die mediale Berichterstattung über Doping, insbesondere Gendoping, analysiert. Dabei sind zwei Aspekte bedeutsam, erstens die Bedeutung medialer Berichte als exogener Faktor und zweitens ihre Nutzung zur Etablierung eines neuen Geschäftsfeldes für Anti-Doping-Organisationen.

3) Werden die theoretischen Überlegungen in ein formales Modell gegossen und die gegenwärtige Situation im Lichte dieses Modells interpretiert. 


\section{Zur sozialen Konstruktion eines Problems - der allgemeine Mechanismus}

Blumer (1971) hat eine indirekte Anleitung geliefert, wie man durch Konstruktion eines sozialen Problems mittelbar die gesellschaftliche Nachfrage nach einer Bekämpfung von als illegitim etikettierten Aktivitäten erhöhen kann und in dessen Verlauf moralische Unternehmer (im Original bei Becker 1966: crusaders) ein Geschäftsmodell entwickeln, das auf Kosten Dritter, oft des Steuerzahlers, etabliert wird (s. dazu auch Klein \& Emrich 2013, Anthonj, Emrich \& Pierdzioch 2013). Blumer (1971) hatte in seinen Überlegungen fünf Phasen bei der Entstehung sozialer Probleme unterschieden und die die Verfestigung des Problems in der sozialen Wahrnehmung und die Institutionalisierung seiner Bearbeitung skizziert.

Die Blumersche Analyse wurde von Schetsche (2000) erweitert und vertieft. Dieser stellt die für die sozialen Wahrnehmungen von Sachverhalten als soziales Problem entscheidenden symbolischen Strukturen und Prozesse in den Mittelpunkt, nämlich den Aufbau des Problemwissens und seine wirksame Verbreitung in Massen- und Netzwerkmedien und entwickelt daraus eine relativistische Theorie, die über ,objektivistische' und ,rekonstruktionistische' Ansätze deutlich hinausgeht und auf fünf Bestimmungen rekurriert.

Soziale Probleme umfassen demnach ,alle (fach-) öffentlichen Thematisierungen, bei denen soziale Akteure Forderungen materieller oder immaterieller Art an gesellschaftliche und staatliche Instanzen stellen ... “ und die die folgenden vier Bedingungen erfüllen: es liegt ein wahrgenommener Verstoß gegen dominierende Werte, schuldlos Geschädigte oder Benachteiligte können benannt werden, die Möglichkeit zur Abhilfe oder Linderung von Not wird als möglich betrachtet, eine moralische Pflicht der Gesellschaft zur Änderung (Besserung) der Zustände wird angenommen.

Die öffentlich im Diskurs identifizierten Problemmuster (Problemname, Erkennungsschema, Prioritätsattribute, Problembeschreibung, Bewertungsmaßstab, Affektauslöser, abstrakte Problemlösung und konkrete Handlungsanleitungen, vgl. Schetsche 2000, S. 130-149) erzeugen abhängig von ihrem Verbreitungsgrad Problemwahrnehmungen, über deren Realitätsgehalt sozial entschieden wird. Je erfolgreicher ein bestimmtes Problemmuster wird, umso wirksamer verdrängt es andere mögliche Problemmuster. Daraus entsteht laut Schetsche (2001, S. 38) ein kollektives „Wahrnehmungskokon“, welches pfadabhängig die Karriere dieses Problems begünstigt und einem stufenförmigen Muster folgt (Schetsche 1996, S. 2137). 
Die im Zusammenwirken von medialer Darstellung und kognitivem Filter dominant gewordene Problemsicht wird durch emotionale Aufladung in den Diskursstrategien nochmals gegen anderslautende Argumente immunisiert und infolge der stets wertrationalen Emotionalisierung (vgl. Opp 1978) nochmals massenmedial verstärkt aufgegriffen und so in den Alltag der Menschen transportiert, wo sie zu Aktionen der Subjekte führt. Emotionalisierung und Agieren der Subjekte erhöhen im Zusammenwirken mit medialer Berichterstattung den Handlungsdruck auf politische Entscheidungsinstanzen, welche dann nach politischer Opportunität entscheiden, wie mit dem Problem umzugehen ist.

Die weitere Verbreitung der Problemmuster als dominanter Deutungsfolie für die Wahrnehmung der Menschen hängt dann von medienspezifischen Konkurrenzprozessen ab, wobei der durch die Emotionalisierung verstärkte Herdentriebeffekt ${ }^{1}$ zu einer Vernachlässigung privater Informationen zugunsten kollektiver führen dürfte. Die Problemwahrnehmungen im Alltag orientieren sich dann maßgeblich an den gelieferten medialen Deutungen und deren Widerspruchsfreiheit.

Für Schetsche (2000, S. 85-99) ist im Sinne seiner relativistischen Problemtheorie die Problemwahrnehmung von folgenden sieben internen und externen Kriterien abhängig: als interne Kriterien nennt er die vollständige Dichotomisierung von Schuld, die Existenz selbstreplikativer Strukturen und das Vorhandensein von Immunisierungsstrategien. Als externe Kriterien nennt er die Herstellung neuer Sinnzusammenhänge, die Kohärenz mit sozial bereits anerkanntem Wissen, die Entstehung institutioneller Deutungspraxen und die Fähigkeit zur Adaption an unterschiedliche Transfermedien. Mit Hilfe dieser sieben internen und externen Kriterien, die er als Konfigurations- und Schnittstellenmerkmale bezeichnet, würden nicht nur die sozialen Karrieren aktueller Probleme im Nachhinein erklärbar, sondern Erfolgschancen zukünftiger Problemwahrnehmungen prognostizierbar. In der fünften Bestimmung differenziert Schetsche (2000) idealtypisch in reale und virtuelle Probleme. Reale und virtuelle Probleme stellen die Eckpunkte eines mehrdimensionalen Kontinuums dar, die dabei lediglich die beiden Extremfälle eines vollständigen bzw. eines gänzlich fehlenden gesellschaftlichen Konsenses über die behaupteten Bedingungen beschreiben. In diesem Sinn sind die Kategorien real und virtuell sozial konstruiert, nehmen also nicht Bezug

1 In ökonomischen Modellen des Herdentriebs geht man davon aus, dass Individuen eigentlich verfügbare private Informationen zugunsten öffentlicher Informationen vernachlässigen und dadurch Gleichförmigkeit im Verhalten entsteht (zu einer Anwendung in einem sportspezifischen Kontext auf die aktuelle Betonung der Vorteile eines dualen Studiums, s. Bischoff, Emrich \& Pierdzioch, 2015). 
z.B. auf die naturwissenschaftlichen oder sozialwissenschaftlichen Grundlagen eines sozialen Problems.

Schetsche untersucht letztlich objektivistische Problemanalysen durch die Untersuchung von Aussagen über diese Bedingungen (Schetsche 2000, S. 165-167). Wie Schetsche (2001, S. 39) ausführt, sind dies „Feststellungen über soziale Bedingungen, die sich in Problemmustern ebenso finden wie in Deutungen, die das jeweils thematisierte Phänomen nicht als soziales Problem definieren. Im Gegensatz zu den bloß „,behaupteten Bedingungen“, von denen die konstruktionistische Theorie spricht, sind diese Sachverhalte dadurch bestimmt, dass sie als real auch von jenen Akteuren angesehen werden, die die Deutung als Problem nicht teilen. Der entscheidende Unterschied zwischen Problemdeutung und gesellschaftlichen Gegendiskursen besteht hier nicht darin, dass in letzteren die Existenz der betreffenden Bedingungen in Zweifel gezogen, sondern dass deren Problematik bestritten wird“.

\section{$3 \quad$ Doping und Dopingbekämpfung als funktionales Element des Spitzensports}

\subsection{Der Normbruch als Instrument der Selbstvergewisserung}

Emile Durkheim (1999) verdanken wir die grundlegende Erkenntnis, dass abweichendes Verhalten bzw. der Normbruch eine positive soziale Funktion hat, was man auf den Sport übertragen kann, und zwar in einer doppelten Perspektive. Einerseits stabilisiert die Sanktionierung des entdeckten Normbruchs die Antidoping-Norm, andererseits ermöglicht die durch nicht entdecktes Doping induzierte und gewissermaßen durch andernorts entdeckte Dopingfälle geadelte Höchstleistung eine erhöhte Nachfrage nach sportlichen Leistungen, wobei der Konsument in seiner asymmetrischen Wissensposition, der für Integrität und Höchstleistung bezahlt hat, zum Zeitpunkt des Konsums nicht weiß, ob die gezeigte Leistung dopingstimuliert war oder nicht (vgl. Emrich \& Pitsch 2009). Zu beachten ist dabei aber auch, dass, obzwar Doping generell die Chance für Höchstleistungen und Rekorde erhöht, damit nicht ausgeschlossen ist, dass besonders talentierte Höchstleister auch ohne Doping Spitzenleistungen erbringen. Der Generalverdacht, dem sie unterliegen, ist dann ein negativer Effekt des Dopings, während die Nachfragestimulanz für Sport und höhere Einkommen durch nicht entdeckte Dopingfälle positive Folgen abweichenden Verhaltens für alle Akteure darstellen.

Popitz (1968) hat generell die besondere Bedeutung des Dunkelfeldes herausgearbeitet. Die präventive Wirkung des Nichtwissens trifft somit auch im Bereich des Dopings zu, erlaubt sie doch vielerlei Spekulationen über das tatsächliche Dopingausmaß, gleichzeitig aber schützt 
die relative Unkenntnis über das wahre Ausmaß der Abweichung die Norm in ihrer Existenz und stabilisiert so den Glauben an die Integrität der gezeigten Leistungen.

\subsection{Zwischen Teststatistik und Thomas-Theorem}

In der jüngeren Vergangenheit konnten zwei empirisch getriebene kritische Richtungen der Analyse der Wirksamkeit von Dopingkontrollen identifiziert werden, und zwar eine teststatistisch getriebene, objektivistische, und eine konstruktivistische, dem ThomasTheorem folgende, wonach dann, wenn Menschen glauben, eine Situation sei real, diese eben auch real in ihren Konsequenzen ist.

Im Sinne der ersten Richtung konnte Pitsch (2009, 2011) zeigen, dass rekurrierend auf das Probleme der Sensitivität und Spezifität bei wiederholten Tests angesichts empirisch ermittelter positiver prädiktiver Werte in einer Population (zur Rate der Doper im Kollektiv, s. Pitsch \& Emrich 2012) bei Anwendung gegenwärtig vorhandener Tests zur Vermeidung falsch positiver eine erhebliche Anzahl falsch negativer Tests auftritt. Dieses objektivistische Argument spielt in der Debatte über Doping und seine Bekämpfung so gut wie keine Rolle, möglicherweise wegen seiner mathematischen Logik, die unkundige Laien eher abschreckt und von kundigen Experten gerne verschwiegen wird, möglicherweise um Einkommenschancen nicht zu gefährden.

Da das vorgenannte Argument kaum diskutiert wird, ist die Abschreckungswirkung von Dopingtests wohl eher eine Folge des Thomas Theorems der Sozialwissenschaften. Athleten glauben offensichtlich mehrheitlich daran, dass Anti-Doping-Tests wirksam sind, weshalb sie insgesamt eine abschreckende Wirkung haben. Wie wird nun dieser Glaube an deren Wirksamkeit genutzt?

In mehreren Beiträgen konnte in der Vergangenheit gezeigt werden, dass Doping und Dopingbekämpfung zu einem integralen Bestandteil einer Sport produzierenden Branche geworden sind. Emrich, Pitsch und Frenger (2013) haben in diesem Kontext die Interessenverschränkungen von Sportlern, Sportverbänden, Kontrollorganisationen, Medien und Politikern analysiert. In einem formalen Modell wurde dann aufbauend auf empirischen Befunden zur Rate von Dopern im Kollektiv bundesdeutscher Spitzensportler (vgl. Pitsch \& Emrich 2012) und aufbauend auf der Quote entdeckter Doper (Adverse Analytical Findings) und den Kosten der Dopingkontrollen das für die internationalen Sportverbände optimale Maß zwischen dopingbelasteter, in Dopingkontrollen nicht entdeckter Höchstleistung und überführten dopenden Höchstleistern modelliert (Frenger, Emrich \& Pitsch 2014).

Konsistent dazu zeigen spieltheoretische Modellierungen, dass gerade die Forderung nach dopingfreiem Sport dazu führt, dass das Bemühen der Veranstalter, dopende Höchstleister des 
Dopings zu überführen, überschaubar bleibt (Büchel, Emrich \& Pohlkamp 2014). Sogar unter der Bedingung, dass es auch Fans gibt, die eher an Dopingsensationen interessiert sind als an ehrlicher Spitzenleistung, bleibt dieser Effekt stabil.

\subsection{Doping und Dopingbekämpfung im Spannungsfeld der Konkurrenz von Nationen}

Emrich und Pierdzioch (2015) wenden theoretische Überlegungen der Volkswirtschaftslehre im Bereich der internationalen Handelspolitik zur Prüfung der Effizienz institutioneller Regelungen zur Antidoping-Politik im Rahmen eines Duopol-Modells an, um zu analysieren, unter welchen Bedingungen in der Konkurrenz um Medaillenanteile bei Olympischen Spielen befindliche Nationen tatsächlich den internationalen Kampf gegen Doping ernsthaft führen. Dazu analysieren sie die Koordination der Anti-Doping-Politik im Verhältnis von WADA zu nationalen NADAs, und können unter Rückgriff auf aus der Zeitinkonsistenztheorie der Volkswirtschaftslehre bekannte Modellierungstechniken belegen, dass die Abwesenheit von formalen Rechtsmitteln zwecks Erzwingung von Kooperationsbereitschaft ein zentrales Problem darstellt, da sich die nationalen Anti-Doping-Politiken in einem Spannungsfeld von nationalen sportlichen Erfolgen, materiellen Ressourcen für die Dopingbekämpfung und der Reputation der Dopingbekämpfer bewegen und es in diesem Spannungsfeld trotz angekündigter Anti-Doping-Programme, ausgelöst durch den internationalen Wettlauf um sportliche Erfolge, zu einer Dopingverzerrung kommt. Es wird also mehr gedopt als bei international koordinierter Antidoping-Politik zu erwarten wäre (für eine detaillierter Übersicht s. die Einleitung in Emrich, Pierdzioch \& Pitsch 2015).

Diese Befunde bedeuten, dass indirekte Subventionierungen der Erfolge der eigenen Nationalmannschaften durch verringerte Kontrollaktivitäten erwartbar sind, wobei zu bedenken ist, dass möglicherweise die Einführung der ersten NADA durch die USA sowohl der Verringerung der Zahl von Dopern in den USA als auch vor allem der Erschwerung der Leistungsproduktion in der UDSSR und DDR sowie anderen Ländern des Ostblocks gedient haben könnte, indem sie entsprechend den sozialen Druck erhöht hat, also Dopingkontrollen als Schaufensternachweis der eigenen Sauberkeit bei gleichzeitiger Erhöhung des sozialen Drucks auf den Systemgegner, der sich dann nur schwer den Kontrollen entziehen kann. Dabei verleiht der First-Movement-Advantage (s. Schumpeters Begriff des temporären Monopolisten) zudem moralische Überlegenheit.

In einem weiteren Beitrag zeigen Emrich und Pierdzioch (2013), dass die Unterstützung für eine internationale wirksam koordinierte Anti-Doping-Politik auf nationaler Ebene durch die jeweiligen nationalen Präferenzen für sportliche Medaillenerfolge und die 
Substitutionsbeziehung sportlicher Erfolge erheblich beeinflusst wird. Diese Substitutionsbeziehung entsteht dadurch, dass jeweils nur eine Nation einen Goldmedaillengewinner in einer bestimmten Disziplin stellen kann. Allerdings können die Athleten des Nichtsiegerlandes immer noch eine Silber- und Bronzemedaille gewinnen, so dass die soziale Rezeption der Substitutionsbeziehung zwischen nationalen sportlichen Erfolgen letztendlich auch von der Wahrnehmung und medial kommunizierten Bedeutung etwa von Medaillenspiegeln abhängen dürfte (vgl. dazu Emrich, Gassmann, Haut, Pierdzioch \& Prohl i. Dr.). Je stärker jedenfalls diese Substitutionsbeziehung ist, desto kleiner wird in dem Modell von Emrich und Pierdzioch (2013) der eh schon begrenzte Möglichkeitenraum für eine internationale Koordination der Anti-Doping-Politik bzw. desto wahrscheinlicher ist es, folgt man Emrich und Pierdzioch (2015), dass es international zu einer Dopingverzerrung kommt. In dem Grenzfall einer sehr intensiven Substitutionsbeziehung muss der Anti-DopingCode der WADA letztlich sogar umfassend die Präferenzen der beteiligten Nationen abbilden, eine kaum lösbare Aufgabe bei divergierenden Präferenzen. Divergierende Präferenzen können entstehen, wenn die Sportler unterschiedlicher Länder in jeweils unterschiedlichen Sportarten erfolgreich sind. Wenn sportliche Erfolge etwa in Ausdauersportarten gefeiert werden, dann könnte die NADA etwa betonen, wie wichtig der Anti-Doping-Kampf gerade in Kraftsportarten ist. Umgekehrt wird bei sportlichen Erfolgen in Kraftsportarten vielleicht gerade die Bedeutung des Anti-Doping-Kampfes in Ausdauersportarten betont. Es liegt dann auf der Hand, dass eine internationale Verständigung auf einen umfassenden Anti-DopingCode unter solchen Bedingungen schwierig ist und folglich die WADA von den NADAs gern für ihre Anti-Doping-Politik kritisiert wird und viele Länder sogar nicht einmal eine NADA etabliert haben (s. dazu Emrich \& Pitsch 2009). Doping und Dopingbekämpfung werden damit letztlich auch durch die Konkurrenz der Nationen um nationalen sportlichen Erfolg zu einem funktionalen Element des Spitzensports, was auf internationalem sportpolitischem Parkett eine systematischen Entkoppelung von Reden und Handeln bedingt (Brunsson 2002), was in eine Problemperpetuierung bei gleichzeitiger Verstetigung der aus der Bearbeitung eben dieses Problems fließenden Einkommensströme mündet.

\section{Doping als Geschäftszweig}

Die Analyse der Kontrollaktivitäten der NADA zeigt eine über die Zeit erheblich steigende Zahl von Dopingkontrollen bei im Wesentlichen gleichbleibendem Verhältnis von Kontrollen und überführten Dopern (s. Tabellen 1a und 1b). 
Tabelle 1a: Zahl der NADA-Kontrollen und Rate der entdeckten Doper 2003 bis $2007^{2}$

\begin{tabular}{crrrrr}
\hline $\begin{array}{c}\text { Zahl der von der WADA } \\
\text { durchgeführten Tests und } \\
\text { Anteil der Adverse }\end{array}$ & 2003 & 2004 & 2005 & 2006 & 2007 \\
Analytical Findings (AAF) \\
(WADA, 2003, 2004, 2005, \\
$\begin{array}{c}\text { 2006, 2007) Year } \\
\text { Zahl der Tests }\end{array}$ & 151,210 & 169,187 & 183,337 & 198,143 & 223,898 \\
AAF & 1.62 & 1.72 & 2.13 & 1.96 & 1.97 \\
\hline
\end{tabular}

Tabelle 1b: Zahl der NADA-Kontrollen und Rate der entdeckten Doper 2008-2012 3

\begin{tabular}{|c|c|c|c|c|c|}
\hline $\begin{array}{c}\text { Zahl der von der WADA } \\
\text { durchgeführten Tests und } \\
\text { Anteil der Adverse } \\
\text { Analytical Findings (AAF) } \\
\text { (WADA, 2008, 2009, 2010, } \\
\text { 2011, 2012) Year }\end{array}$ & 2008 & 2009 & 2010 & 2011 & 2012 \\
\hline Zahl der Tests & 274.615 & 277.928 & 258.267 & 243.193 & 267.645 \\
\hline AAF & 1,84 & 2,02 & 1,87 & 2 & 1,76 \\
\hline
\end{tabular}

Ein Subunternehmer der NADA, die Firma PWC ${ }^{4}$, die für die NADA die Dopingkontrollen durchführt, hat im Zuge der steigenden Zahl von Kontrollen im Verlauf der Jahre 2005 bis 2012 ihre Bilanzsumme in etwa vervierfacht (s. Abbildung 1). Einem Auftraggeber, NADA, stand faktisch längere Zeit ein Auftragnehmer, die Firma PWC, gegenüber. Gewöhnlich

2 Seit 2012 führt die NADA zusätzlich Pferdekontrollen für Doping durch (http://www.nada.de/fileadmin/user_upload/nada/Downloads/Jahresberichte/NADA_Jahresbericht_2012_kl.p df). Die Quote der entdeckten Dopingfälle (AAF) bleibt auch in den Jahren nach 2007 im Wesentlichen konstant.

Die NADA wird argumentieren, dass gerade wegen der vielen Kontrollen nicht mehr Doper entdeckt werden, weil eben die Abschreckung so gut funktioniert. Auffällig ist auch die Parallelität zum Gesundheitssektor allgemein. In allen anderen Sektoren senkt technischer Fortschritt die Kosten, nur im Gesundheits- und Sportsektor scheint der Fortschritt die Kosten zu treiben.

Seit 2012 führt die NADA zusätzlich Pferdekontrollen für Doping durch (http://www.nada.de/fileadmin/user_upload/nada/Downloads/Jahresberichte/NADA_Jahresbericht_2012_kl.p df). Die NADA wird argumentieren, dass gerade wegen der vielen Kontrollen nicht mehr Doper entdeckt werden, weil eben die Abschreckung so gut funktioniert. Auffällig ist auch die Parallelität zum Gesundheitssektor allgemein. In allen anderen Sektoren senkt technischer Fortschritt die Kosten, nur im Gesundheits- und Sportsektor scheint der Fortschritt die Kosten zu treiben.

4 S.http://www.nada.de/de/doping-kontroll-system/zusammenarbeit-mit-pwc/\#.VUOyk0k2WBE 
münden solche quasi bilateralen Monopole in der Aushebelung von Marktwirkungen und eröffnen diskretionäre Spielräume. Die NADA beauftragte dann zur Durchführung der Dopingkontrollen seit 2015 zusätzlich das international tätige Unternehmen IDTM (International Doping Tests \& Management; http://idtm.se/). ${ }^{5}$ IDTM ist ein Dienstleister für die Durchführung von Dopingkontrollen mit Hauptsitz in Schweden und verfügt über ein globales Netz von Dopingkontrolleuren. Für die NADA kommen deutschsprachige Kontrolleure zum Einsatz. Alle zu kontrollierenden Athleten wählt ausschließlich die NADA aus, die Probennahme und der Probentransport zu den WADA akkreditierten Laboren in Köln und Kreischa erfolgen durch die Auftragnehmer. ${ }^{6}$

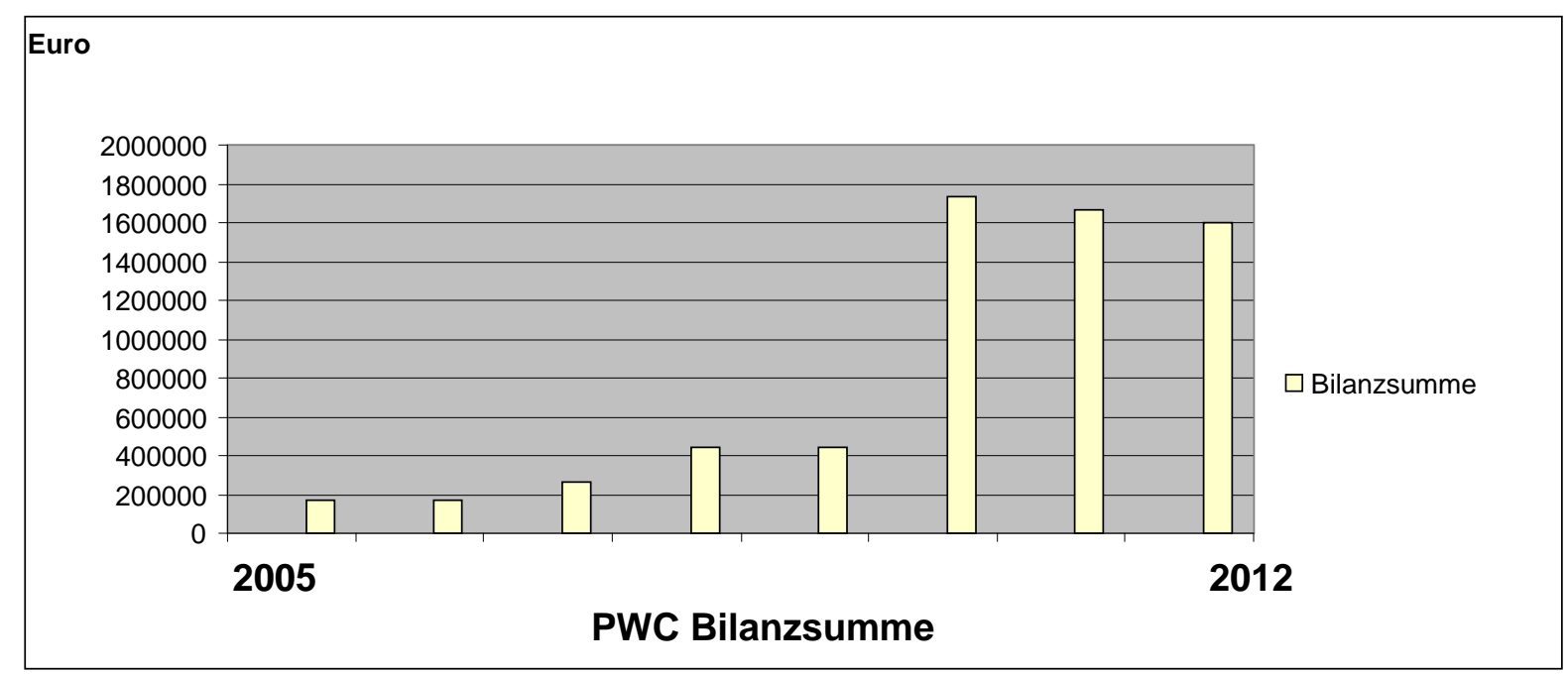

Abbildung 1: Offiziell ausgewiesene Bilanzsummen der Firma PWC von 2005 bis 2012

Wir stellen somit in einem Zwischenfazit fest: Die Zahl der Kontrollen ist kontinuierlich gestiegen, die Subunternehmer haben ihre dopingbezogenen Umsätze stark erhöht, die Quote der entdeckten Doper aber ist in etwa gleichgeblieben. Erklärt wird dies mit dem Hase und Igel-Phänomen, laut dem der clevere Dopingkonsument dem Kontrolleur immer einen Schritt

5 Zu diesem Zweck hatte die NADA die Durchführung der Dopingkontrollen ab 2015 nach europäischem Vergaberecht öffentlich ausgeschrieben. Den Zuschlag erhielten die Firma Professional Worldwide Controls GmbH (PWC) und die Firma IDTM. Neben PWC und IDTM ist weiterhin das Unternehmen Global Quality Sports GmbH (GQS) für die Durchführung eines Teils der Wettkampfkontrollen der NADA im Jahr 2015 zuständig. Die öffentliche Auftragsbekanntmachung sowie die Auftragsvergabe sind nachzulesen unter http://ted.europa.eu/udl?uri=TED:NOTICE:5663-2015:TEXT:DE:HTML\&tabId=1. Zur wenig aussagekräftigen Ausschreibung des Auftrags, s. Deutschland-Bonn: Dienstleistungen von medizinischem Personal, 2015/S 005-005663, Bekanntmachung vergebener Aufträge, Dienstleistungen http://ted.europa.eu/udl?uri=TED:NOTICE:5663-2015:TEXT:DE:HTML\&tabId=1

6 S. http://www.nada.de/de/doping-kontroll-system/zusammenarbeit-mit-idtm/\#.VUOzOkk2WBE. 
voraus sei und als Antwort auf diese Herausforderung werden immer mehr Kontrollen und damit immer mehr Ressourcen gefordert. ${ }^{7}$ Es liegt allerdings auch der Verdacht nahe, dass sich hier ein stabiler Zustand herausgebildet hat, der der Institution Sport im internationalen Sinn, also auf der Ebene des olympischen Sports, optimale Einkommenschancen angesichts erkennbarer Zielkonflikte sichert.

\section{Die Logik der Medien}

Medien berichten über Doping, und zwar sowohl informierend als auch etikettierend (vgl. Philipp 2002, aktuell dazu am Beispiel der Leichtathletik und der Tour de France Frenger, Emrich \& Klein i. Dr.). Dabei produzieren negative Wertungen zumindest anfänglich höhere Aufmerksamkeitswerte. Würde jedoch konsequent und auf hohem Niveau negativ berichtet und dabei gemäß der massenmedialen Logik (vgl. Franck 1998) auch entsprechend in den negativen Wertungen übertrieben und dies ohne konjunkturelle Schwankungen in den bevorzugten Inhalten der Presseberichte und der Art ihrer Darstellung durchgehalten, würde sich wahrscheinlich durch Sättigungseffekte ebenso wie durch enttäuschte Gefühle begeisterter Fans die Nachfrage reduzieren, obwohl ein Teil des Publikums auch am Unterhaltungswert der Abweichung und ihrer medial kommentierten Entdeckung besonders interessiert sein mag. In diesem Kontext stimulieren mediale Meldungen über Gendoping nicht nur die Nachfrage nach Medienberichten, sondern sie liefern auch in hohem Maße Anschlusskapazitäten für moralische Unternehmer.

Einerseits ist das Thema Gendoping beim Menschen von jeher seit der Antike mit ihren Chimären mit diffusen Ängsten besetzt, was einerseits eine Art gesellschaftlicher Gänsehaut erzeugt (zu den Mechanismen von Moral Panic s. Walby \& Spencer 2011), andererseits aber erzeugt genau diese Gänsehaut Nachfrage nach medialen Informationen und Meldungen. Weitgehend irrelevant für diesen konstruktivistischen Prozess ist der objektivistische Befund z.B. von Wells (2015, S. 151ff, hier S. 154), der klar darauf verweist, dass die vorwiegend an Mäusen gewonnenen Befunde etwa zum extremen Muskelwachstum durch genetische Manipulation keineswegs uneingeschränkt auf Menschen übertragbar seien. Zwar konnte an

7 Dies erinnert an die Polizei. Auch sie macht permanent auf ihre Erfolge in der Verbrechensbekämpfung aufmerksam und legitimiert so ihre Arbeit. Gleichzeitig fordert sie permanent neue Kräfte, um die trotz ihrer erfolgreichen Arbeit steigende Verbrechenszahl wirksam zu bekämpfen. 
Mäusen und nicht menschlichen Primaten z.B. ein EPO-Effekt durch genetische Manipulation erzeugt werden, aber auch hier ist die Übertragbarkeit keineswegs gesichert. ${ }^{8}$

In den diffusen Vorstellungen verschiedener Akteure, vornehmlich der relativen medialen Laien, entwickelt sich so durch mediale Berichte und die Logik ihrer Nachfrage das Bild einer bedrohten Zukunft des Sports, in der künstlich hergestellte genetische Sonderheiten den sportlichen Erfolg prägen und das Humane und Natürliche bedrohen. Während Teile der Wissenschaft dies durchaus kritisch sehen, verstärken andere diese bedrohlichen Szenarien aktiv. Dabei wirken antizipierte Drittmittelmöglichkeiten als Anreiz für das Tätigen und verbale „Aufrüsten“ affirmativer Aussagen (vgl. zu diesen Prozessen im Wissenschaftsbetrieb grundlegend Emrich \& Pierdzioch 2011). Insgesamt ist dieser Prozess noch mitten in der Phase der identifizierten Problemmuster (Problemname, Erkennungsschema, Prioritätsattribute, Problembeschreibung, Bewertungsmaßstab, Affektauslöser, abstrakte Problemlösung und konkrete Handlungsanleitungen, vgl. Schetsche 2000, S. 130-149), die abhängig von ihrem Verbreitungsgrad Problemwahrnehmungen erzeugen, über deren Realitätsgehalt entschieden wird.

Wie sieht nun die emotional aufgeladene und gewöhnlich negativ wertende mediale Berichterstattung über das Phänomen Gendoping aus? Betrachtet man ausgewählte Pressemeldungen im Zeitraum von 2010 bis 2015, ist eine relativ einheitliche Tendenz zu erkennen. So werden seit 2013 auf der Verbotsliste der WADA unter Gendoping die Übertragung von Nukleinsäuren, also DNA oder RNA, und die Anwendung normaler oder genetisch veränderter Zellen genannt. Als echte Form des Gendopings gelten allerdings nur Methoden, die für Patienten mit genetisch bedingten Erkrankungen gedacht sind und die sich allesamt mit Ausnahme einer einzigen für den Menschen zugelassene Gentherapie, die eine sehr seltene Krankheit des Fettstoffwechsels heilen soll, noch im Tierversuchsstadium befinden (Brodmerkel 2015, s. auch Müller-Junge 2014). Als wahrscheinlichster Kandidat für künftiges Gendoping wird von Experten Follistatin genannt, das Muskelwachstum stark anregt. Man befürchtet, dass das bei Affen beobachtete Muskelwachstum nach klinischer Bestätigung beim Menschen auch dort Anwendung finde, allerdings, bisher steht eine solche Studie noch aus (Ballwieser 2012). Im Interview mit einem Anti-Doping-Experten in TOnline vom 02.03.2010 wird auch klar ausgeführt, dass er nicht sieht, dass Gendoping erfolgreicher sein kann als beispielsweise Testosteron. An anderer Stelle heißt es zum selben

8 Dies gilt im Übrigen auch für den therapeutischen Einsatz der Gentherapie. Wells (2015, S. 154f.) schreibt unter Bezug auf das Journal of Gene Medicine (2014) „Although there have been 1.892 human gene therapy trials approved worldwide between 1989 and 2013 very few have shown clear efficacy." 
Sachverhalt, dass niemand wisse, „ob die Grenze zwischen Tierversuch und menschlichem Experiment irgendwo auf der Welt bereits überschritten wurde" (Apfel 2012), aber die drohenden Gefahren werden dessen ungeachtet als für den Athleten kaum beherrschbar, die Veränderungen als unwiderruflich bezeichnet, weshalb die WADA die direkte Veränderung von Genen bereits 2004 vorsorglich verboten hat. Unter Bezug auf einen Doping-Experten wird betont, dass man ,jetzt“ (gemeint ist hier das Jahr 2012, die Verf.) die Grundlagen schaffen müsse, um auf mögliche Entwicklungen vorbereitet zu sein. Spiller (2012) erweitert dieses Bedrohungsszenario und betont die Gefährdung breiter Bevölkerungskreise durch gentechnische Manipulationen insbesondere von Laien. In der Pharmazeutischen Zeitung online (45/2010) wird im Kontext des Gendopings darauf hingewiesen, dass die WADA die Forschung zur Entwicklung von Nachweisverfahren für Gendoping für zwei Forscher allein in den Jahren 2006 bis 2010 mit 980.000 Dollar unterstützt hatte, obwohl völlig unklar war und ist, inwieweit im Profisport Gendoping überhaupt angewendet wird. 2013 wird dann laut Handelsblatt vom 15.05.2013 der Radsportler Ubeto wegen Gendopings von seinem Arbeitgeber suspendiert. Vorgeworfen wurde dem Radsportler, dass er eine unter der Bezeichnung GW 501516 bekannte Substanz eingenommen habe, mit deren Hilfe Leistungssportler gleichzeitig die für Ausdauerleistungen relevante Muskulatur aufbauen und Gewicht reduzieren können, wobei der Vorwurf des Gendopings angesichts der Suspendierung durch seinen Arbeitgeber völlig ungeklärt bleibt.

Je erfolgreicher medial ein bestimmtes Problemmuster entsteht, umso wirksamer verdrängt es andere mögliche Problemmuster. Inwieweit aus der Debatte um Gendoping ein kollektiver „Wahrnehmungskokon“ entsteht, der pfadabhängig die Karriere dieses Problems begünstigt (Schetsche 1996, S. 21-37) ist noch offen. Am Ende des Prozesses könnte jedoch Gendoping als anerkanntes Problemfeld von Dopingaktivitäten stehen, welches gezielt bekämpft wird.

Ein Blick auf das Interesse an dem Google-Schlagwort „Gendoping“ ergibt einen ersten Eindruck, welche Karriere dieser Begriff in der Vergangenheit gemacht hat. Abbildung 2 zeigt die starke Nachfrageerhöhung nach dem Phänomen Gendoping im Kontext Olympischer Spiele. 


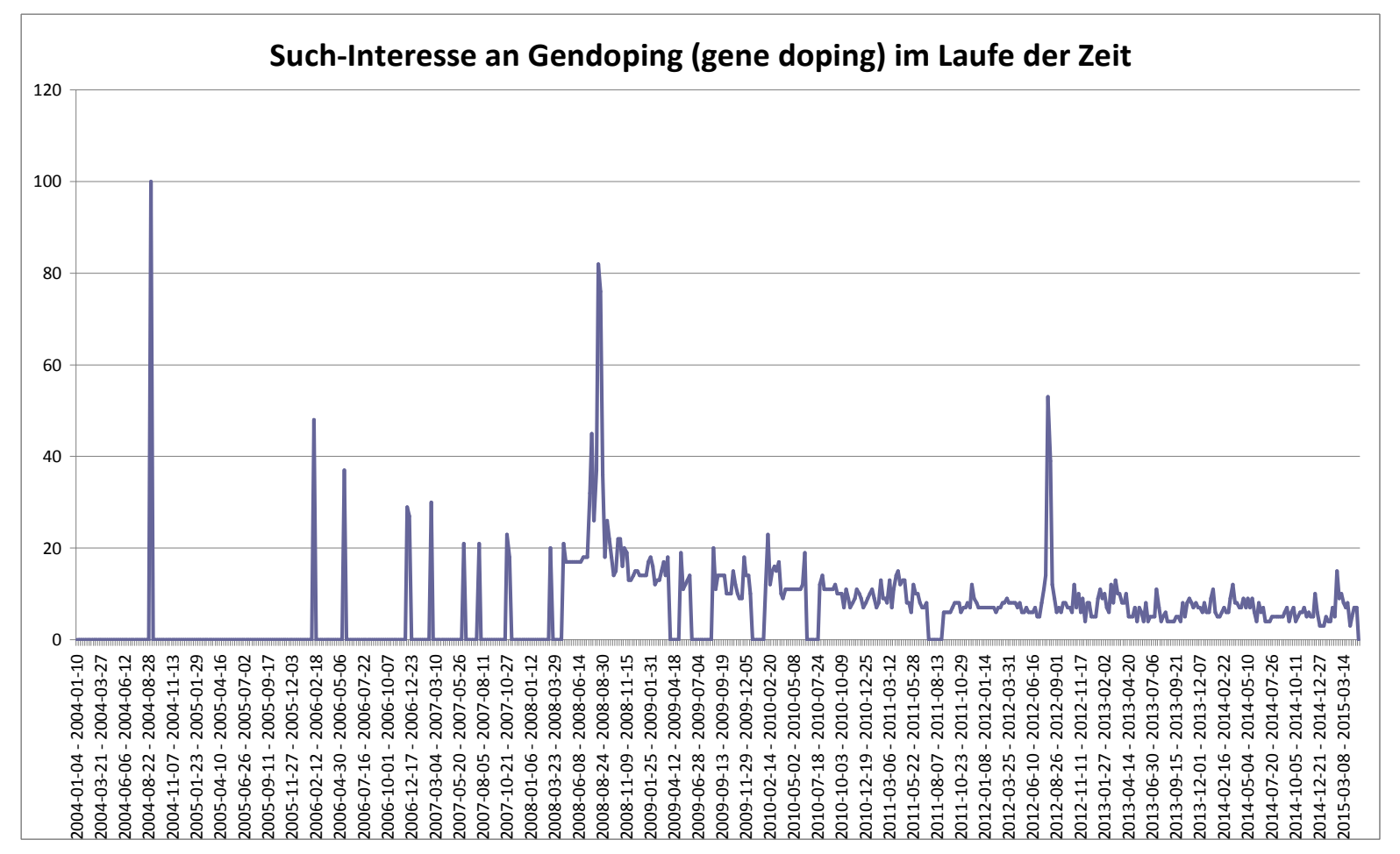

Abbildung 2: Suchinteresse am Schlagwort Gendoping im Zeitverlauf

Abbildung 3 verdeutlicht die in Deutschland wesentliche ausgeprägtere Suchaktivität im Vergleich zu Italien, Brasilien und den Vereinigten Staaten von Nordamerika und verweist damit auf mögliche kulturelle Unterschiede im Bereich der Dopingproblematik.

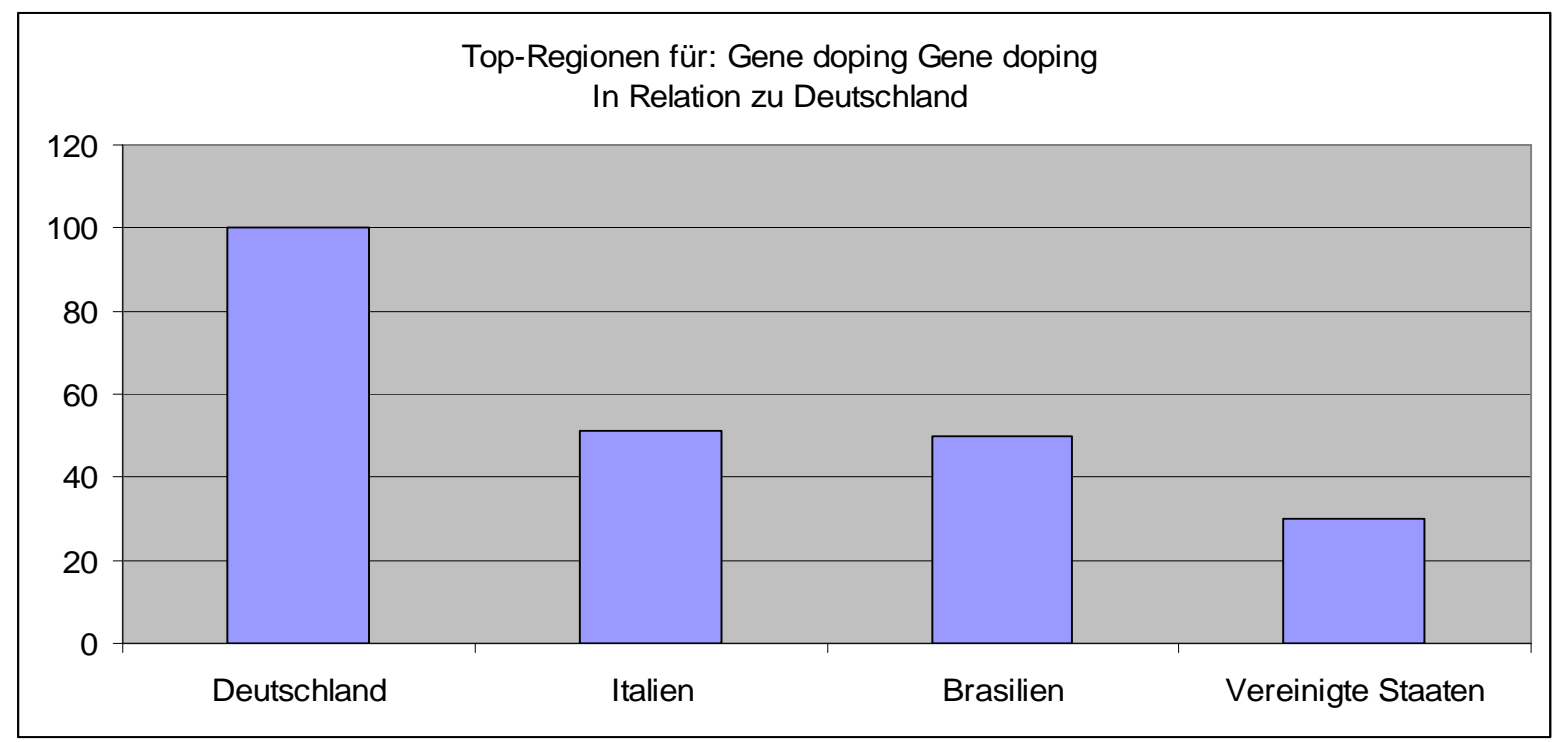

Abbildung 3: Suchverhalten zum Begriff Gendoping im Zeitverlauf. Google Abfrage vom 28.4.2015 


\section{Sozioökonomische Modellbildung}

Doping wird in der Regel mit Instrumenten der Spieltheorie (Breivik 1992, Büchel et al. 2015, Haugen 2004, Eber 2008, und andere) analysiert, vereinzelt kommen auch Methoden der politischen Ökonomik zum Einsatz (z.B. Eber 2002, Emrich \& Pierdzioch 2013, 2015, Emrich, Pierdzioch \& Oestmann i. Dr.).

Hier soll ein anderer Ansatz gewählt werden und Doping aus der Sicht einer für die Bekämpfung des Dopings zuständigen Institution unter Einsatz eines auf der Umweltökonomik aufbauenden Modellierungsansatzes beleuchtet werden (für eine Lehrbuchdarstellung einschlägiger umweltökonomischer Modelle, vgl. Perman, Ma, McGilvray, Common \& Maddison 2011). Der Rückgriff auf Erkenntnisse der Umweltökonomik bietet sich an, weil aus der Sicht einer Kontrollinstitution dopende Athleten quasi eine nachwachsende natürliche Ressource sind. Das Durchführen von DopingKontrollen ist in dieser Sicht dann einfach ein einkommengenerierender Erntevorgang. Das Problem der Kontrollinstitution ist folglich, Doping-Kontrollen derart einzusetzen, dass die Population dopender Athleten zwar bearbeitet, aber eben nicht komplett ausgelöscht wird, weil andernfalls der Einkommensstrom versiegen würde.

Um die grundsätzliche Modellierungsidee zu verdeutlichen, soll nachfolgend ein stilisiertes Modell entwickelt werden. Da das Modell nicht auf einen bestimmten Datensatz kalibriert werden soll, wählen wir bewusst einfache funktionale Zusammenhänge für die mathematische Modellierung. So gehen wir davon aus, dass Veränderungen im Bestand dopender Sportler durch die nachfolgende lineare Bewegungsgleichung abgebildet werden:

$\dot{D}=g D-s T$,

wobei $D$ die Population der dopenden Sportler, $T$ die Anzahl der durchgeführten DopingTests, $g>0$ die Wachstumsrate der Population ist und der Parameter $s>0$ für die Verlässlichkeit der Doping-Tests (vgl. die Anmerkungen zu Sensitivität und Spezifität in Abschnitt 3.1) steht. Der Punkt über einer Variablen steht für die Ableitung nach der Zeit, wobei zur Vereinfachung der Notation auf den Ausweis eines Zeitindex verzichtet wird.

Die Annahme einer konstanten Wachstumsrate erscheint auf den ersten Blick wenig plausibel und in der Tat könnte man z.B. die Frage diskutieren, in welchem Umfang das System Sport dopende Sportler verkraften kann und ob es in diesem Sinne eine natürliche Grenze für das Wachstum der Population dopender Sportler gibt. Der hier postulierte lineare Zusammenhang erscheint dann verständlicher, wenn berücksichtigt wird, dass Doper aus der Sicht der Kontrollinstanz nachwachsen, also in jeder neuen Athletengeneration ein bestimmter Anteil der Sportler zu Doping-Substanzen greift. Wenn gleichzeitig Doping-Proben aufbewahrt 
werden und auch z.B. nach dem Karriereende, wenn neuere Testverfahren entwickelt worden sind, reanalysiert werden können, dann ist eine lineare Wachstumsgleichung zumindest als erste Approximation plausibel. Wie auch immer, hier soll es um die Illustration der grundlegenden Modellierungsidee gehen und daher belassen wir es bei der linearen funktionalen Form.

Wie nun sollte die Kontrollinstanz die Population dopender Sportler bewirtschaften? Die Antwort auf diese Frage hängt natürlich von der Zielfunktion der Kontrollinstanz ab. Zur Konstruktion der Zielfunktion unterstellen wir, dass das Budget (sofern es sich eine staatliche Einrichtung handelt) bzw. der Gewinn (sofern es eine privatwirtschaftliche Institution mit dem Absicht der Gewinnerzielung handelt) von zwei Faktoren abhängt:

1) Einerseits wird die Anzahl der durchgeführten Doping-Tests eine Rolle spielen, wobei wir postulieren, dass das Budget bzw. die Gewinne eine linear ansteigende Funktion der Anzahl der durchgeführten Tests ist.

2) Andererseits wird das mediale und politische Problembewusstsein eine Rolle spielen.

Mit anderen Worten, wenn Gendoping als Problem eingeschätzt wird, dann wird die Kontrollinstanz zusätzliche Mittel einwerben können, um z.B. Forschungsprojekte durchzuführen oder durchführen $\mathrm{zu}$ lassen. Diesbezüglich unterstellen wir einen quadratischen Zusammenhang, weil gewissermaßen die Ausdehnung der Kontrollaktivitäten auf neue Geschäftsfelder als ein zentraler Werttreiber identifiziert wird. Wir postulieren:

$$
B=a T+\frac{1}{2} c D^{2} \text {, }
$$

wobei $B$ für das Budget der Kontrollinstitution steht. Der Parameter $a>0$ kann als Stückpreis (oder alternativ Stückgewinn) interpretiert werden. Der Parameter $c>0$ steht dafür, in welchem Umfang Doping allgemein und neue vermutete Phänomene wie Gendoping budgetwirksam instrumentalisiert werden können. Wir unterstellen hier, dass diese Instrumentalisierungskapazität durch die Population dopender Sportler bestimmt wird, wobei letztere das wahrgenommene bzw. vermutete Bedrohungspotential des Doping-Phänomens approximiert.

Wenn wie Fall des Gendopings über ein Problem diskutiert wird, welches zur Zeit selbst nach Auskunft der Experten, die darüber mit Mitteln der Anti-Doping-Organisationen forschen, überhaupt nicht bzw. noch nicht existiert, so kann Gleichung (2) so interpretiert werden, dass historische Erfahrungen mit „konventionellem“ Doping im Doping-Diskurs auf das neue Phänomen übertragen werden und als Folge der medialen Krisenkonstruktion und dem dadurch ausgelösten Organisations-Stress (s. zu Organisationsstress allgemein Brunsson, 
2002), der einhergeht mit höherem Legitimationsdruck, die Kontrollinstitution mehr Ressourcen auf sich vereinen kann.

Bei intertemporaler Betrachtung maximiert die Kontrollinstitution das Integral über ihre (Perioden-) Zielfunktion:

$\max \int_{0}^{\infty} B e^{-r t} d t$

wobei $r>g$ ein Diskontierungsfaktor ist.

Da das vorliegende intertemporale Optimierungsproblem linear in der Entscheidungsvariable der Kontrollinstitution ist, ergibt sich eine einfache Lösung. Die Lösung kann abgeleitet werden, indem z.B. die Euler-Gleichung der Variationsrechnung ermittelt wird. Die EulerGleichung ergibt:

$D=(r-g) \frac{a}{s c}$

und demzufolge gilt $\dot{D}=0$, weshalb aus Gleichung (1) unmittelbar gefolgert werden kann:

$T=(r-g) \frac{g a}{s^{2} c}$.

Die Kontrollinstanz passt somit die Kontrollaktivitäten unmittelbar auf ihr optimales Niveau an. Dieses Niveau hängt positiv ab von der (per Annahme positiven) Differenz zwischen dem Diskontierungsfaktor und der Wachstumsrate der Doper-Population und von der „Budgetmarge“ $a$. Je größer diese Marge ist, desto mehr Tests werden durchgeführt. Der Parameter $c$ wiederum steht in einem inversen Verhältnis zu den Kontrollaktivitäten, denn Geschäftsfelder müssen zunächst wachsen, damit sie anschließend bearbeitet werden können. Des Weiteren hängt der Umfang der Kontrollaktivitäten invers von der Verlässlichkeit der Doping-Tests, $s$, ab. Wenig leistungsstarke Tests müssen also besonders häufig eingesetzt werden, um die Population der Doper auf dem aus der Sicht der Kontrollinstitution optimalen Niveau zu halten.

\section{Diskussion und Ausblick}

Medial konstruierte Krisen können als „soziales Schmiermittel“ und konjunktureller Treiber der Nachfrage nach Anti-Doping-Aktivitäten und damit als Mittel der Erzielung von Einkommen für die genannten Akteure dienen, und zwar um so mehr, je emotionaler aufgeladen die öffentliche Atmosphäre ist und je bedrohlicher Doping empfunden wird. Trittbrettfahrende moralische Unternehmer bleiben in einem solchen Kontext nicht aus.

Mediale Berichte, zumal wenn sie emotional aufgeladen sind, bedrohen die Legitimität des Spitzensports, der in der Folge die öffentliche Unterstützung riskiert. Es kann deshalb höchst 
rational sein, das medial konstruierte Problem demonstrativ aufzugreifen und seine Bearbeitung vor allem auf Kosten des Steuerzahlers an Kontrollorganisationen zu delegieren Politiker und Sportfunktionäre erweisen sich damit als sozial verantwortliche Entscheider und erhöhen die Chancen auf eine verbesserte eigene Reputation.

Insofern entwickelt sich nicht selten eine Art Doppelbindungsprozess zwischen Akteuren in Sportorganisationen, Medienvertretern und den Kontrollagenturen. Außerdem gilt grundsätzlich das negative Kassandra-Syndrom. Man warnt vor etwas. Tritt es ein, nun gut, man hat ja davor gewarnt, tritt es nicht ein, auch gut, man hat ja davor gewarnt (Hüttl 1998). An dieser Stelle wirken Wissenschaftler, die erst einmal die Existenz des Explanandums untersuchen wollen, unter Umständen sogar hemmend für die Pläne der Entscheider in Sportorganisationen und in Kontrolleinrichtungen. In beiden ist man ebenso wie in der Politik allgemein nicht zuletzt aufgrund der Wahlperioden an schneller Umsetzung und Entwicklung von Programmen zur Bearbeitung des tatsächlichen oder vermeintlichen Problems interessiert, die dann wiederum medial kommuniziert werden und Entschlossenheit der Entscheider dokumentieren. Wissenschaftler wirken bisweilen auch an diesem Prozess mit, sofern sie, aus welchen Gründen auch immer, rasche Erfolge in Form von Publikationen und eingeworbenen Drittmitteln erzielen möchten und dafür die Existenz des Explanandums, obgleich keineswegs gesichert, in ihrer Forschung einfach voraussetzen. Sie finden sowohl auf Ebene der Sport- als auch der Kontrollorganisationen Mitstreiter. Denkbar ist, dass auf diesem Weg Programme oder Maßnahmen zur Bearbeitung eines sozialen Problems „Karriere machen“, die keineswegs in ihrer Wirkung als gesichert betrachtet werden können. Sie bedienen dann gleichzeitig das Drittmittelinteresse der Forscher, markieren die soziale Verantwortung der Entscheider und beide dienen der politischen Entscheidungsebene als öffentlich sichtbarer Beleg dafür, dass ein soziales Problem des Sports entschieden bekämpft wird. Also nicht nur ein Double Bind, sondern ein Triple Bind, der begleitet wird von einem Agent Provocateur, der den Prozess anheizt und selbst davon profitiert, nämlich den Medien. Die wiederum erzielen nur Aufmerksamkeit, wenn sie mit ihren Bedrohungsszenarien genauso übertreiben, dass man es gerade noch glauben kann, wozu wiederum Wissenschaftler die „Information“ liefern, die dann die Entscheidungsträger in Organisationen entweder getrieben von der öffentlichen Darstellung und/oder aus Eigeninteresse aufgreifen. Diese drängen dann die Politik zur Ressourcenmobilisierung oder werden von ihr nach medialem Druck zur intensiveren Bearbeitung unter Bereitstellung von Ressourcen genötigt, also eine zukunftssichere Branche. 


\section{Literatur}

Anthonj, P., Emrich, E.und Pierdzioch C. (2013): Gewalt und Gewaltbekämpfung im deutschen Fußball Empirische Bestandsaufnahme und sozioökonomische Modellbildung. Diskussionspapiere des Europäischen Instituts für Sozioökonomie e.V. Nr. 1, 2013.

Apfel, P. (2012): Gen-Doping: Der unsichtbare Betrug mit den Sport-Rekorden FocusOnline

01.08.2012 (http://www.focus.de/gesundheit/gesundleben/fitness/leistung/tid-26651/olympia2012-in-london-gen-doping-der-unsichtbare-betrug-mit-den-sportrekorden_aid_786726.html)

Ballwieser, D.: Gendoping: Lebensgefährliches Spiel mit dem Erbgut - SpiegelOnline 09.08.2012 (http://www.spiegel.de/gesundheit/ernaehrung/gendoping-sportlerwuerden-ihr-leben-ohne-nutzen-gefaehrden-a-848689.html)

Becker, H.S. (1966): Outsiders: Studies in Sociology of Deviance, New York / London. Bischoff, F., Emrich, E. und Pierdzioch, C. (2015): Berufsakademien zwischen Fakten und Fiktionen: Eine empirische Analyse anhand eines Beispiels aus dem Sportund Fitnessbereich, Zeitschrift für Evaluation, 14, 83-103.

Blumer, H. (1971): Social Problems as Collective Behavior, Social Problems, 18, 298306.

Breivik, G. (1992): Doping Games. A Game Theoretical Exploration of Doping, International Review for the Sociology of Sport, 27, 235-255.

Brodmerkel, A. Auf Siegeskurs mit fremden Genen - Berliner Zeitung 12.01.2015 (http://www.berliner-zeitung.de/wissen/gendoping-im-sport-auf-siegeskurs-mitfremden-genen,10808894,29547364.html)

Brunsson, N. (2002): The Organization of Hypocrisy: Talk, Decisions and Actions in Organisations, Copenhagen.

Büchel, B.; Emrich, E. und Pohlkamp, S. (2014): Nobody's Innocent: The Role of Customers in the Doping Dilemma, Journal of Sports Economics, first published on October 7, 2014 as doi:10.1177/1527002514551475

Doping - "Die Sportler nehmen in Kauf, nicht so lange zu leben" Interview mit Perikles Simon - T-Online (http://www.t-online.de/sport/id_21651478/ gendoping-perikles-simon-spricht-ueber-den-einsatz-und-die-gefahren.html)

Durkheim, E. (1999, orig. 1895): Die Regeln der soziologischen Methode, 4. Aufl., Frankfurt am Main: Suhrkamp. 
Eber, N. (2002): Credibility and Independence of the World Anti-Doping: A BarroGordon-Type Approach to Antidoping Policy, Journal of Sports Economics, 3, 90-96.

Eber, N. (2008): The Performance-Enhancing Drug Game Reconsidered: A Fair Play Approach. Journal of Sports Economics, 9, 318-327.

Emrich, E. und Pitsch, W. (2009): Zum Dopingkontrollmarkt - Sind Investitionen in den Anschein von Ehrlichkeit lohnender als die Ehrlichkeit selbst? In: Emrich, E.; Pitsch, W. (Hrsg.): Sport und Doping. Zur Analyse einer antagonistischen Symbiose. Frankfurt am Main u.a.: Lang, 111-130.

Emrich, E., Frenger, M. und Pitsch, W. (2013): Soziologische und ökonomische Funktion von Grenzwerten - eine kurze Skizze, Diskussionspapier Nr. 4, Europäisches Institut für Sozioökonomie.

Emrich, E. und Pierdzioch, C. (2013): Joining the International Fight Against Doping, Applied Economics Letters, 20, 1379-1382.

Emrich, E. und Pierdzioch, C. (2015): A Note on the International Coordination of Antidoping Policies, Journal of Sports Economics, 16, 312-321.

Emrich, E., Pierdzioch, C. und Pitsch, W. (2015): Falsches Spiel im Sport. Analysen zu Wettbewerbsverzerrungen, Saarbrücken: universaar.

Emrich, E., Gassmann, F. Haut, J., Pierdzioch, C. und Prohl, R (i. Dr.): Medaillen für die nationale Repräsentanz? Zur Bedeutung von Medaillenerfolgen bei Olympischen Spielen. Angenommen zur Publikation für Sport und Gesellschaft. Heft 2. 2015.

Emrich, E., Pierdzioch, C. und Oestmann, M. (im Druck): A Note on Persistent National Olympic Success and Time-Inconsistent Anti- Doping-Policy. In G. Schafmeister und S. Dallwig (Hrsg.), Evolution und Revolution in der Sportwirtschaft. Perspektiven des Wandels aus sportökonomischer Sicht, Schorndorf: Hofmann-Verlag.

Franck, G. (1998): Ökonomie der Aufmerksamkeit. Ein Entwurf, München / Wien.

Frenger, M., Emrich, E. und Pitsch, W. (2014): How to Produce the Belief in Clean Sports Which Sells, Performance Enhancement \& Health, Published Online 22 October 2014, DOI: 10.1016/j.peh.2014.09.001

Frenger, M., Emrich, E. und Klein, M. (in Druck): Mediale Aufbereitung und Bewertung von Dopingfällen in den Printmedien. In G. Schafmeister \& Ellert u.a. 
(Eds.), Sportökonomie: Vol. 16. Evolution und Revolution in der Sportwirtschaft. Perspektiven des Wandels aus sportökonomischer Sicht. Schorndorf: Hofmann.

Gendoping: Lampre suspendiert Ubeto - Handelsblatt vom 15.05.2013 (http://www.handelsblatt.com/radsport-doping-gendoping-lampre-suspendiertubeto/8213076.html)

Gendoping: Nachweis durch Bluttest möglich - Pharmazeutische Zeitung online 45/2010 (http://www.pharmazeutische-zeitung.de/index.php?id=35884)

Hayek, F. A. von (1969): Bemerkungen über die Entwicklung von Systemen von Verhaltensregeln. In Friedrich A. von Hayek (Hrsg). Freiburger Studien. Gesammelte Aufsätze. Tübingen: Mohr 1969, 155-160.

Hüttl, R. (1998): Debatte: Das negative Kassandra-Syndrom. Wissenschaft im Streit. Berlin-Brandenburgische Akademie der Wissenschaften. Interdisziplinäre Forschungsgruppen, Juni 1998, verfügbar unter http//www.bbaw.de/termine/1998/11/26_text.html Zugriff am 17.06.2005

Joachim Müller-Jung, J. (2014): Therapie oder Anleitung zum Gendoping? Muskeln aus dem Jungbrunnen - FAZ 17.02.2014 (http://www.faz.net/aktuell/wissen/ therapie-oder-anleitung-zum-gendoping-muskeln-aus-dem-jungbrunnen12806972.html)

Klein, M. und Emrich, E. (2013): Konstruktion sozialer Probleme. Zur Rolle von moralischen Kreuzfahrern im wissenschaftlichen Legitimationsprozess, sozialersinn, 14, 103-121

Opp, K.-D. (1978): Theorie sozialer Krisen: Apathie, Protest und kollektives Handeln, Hamburg.

Perman, R., Ma, Y., McGilvray, J., Common, M.S. und Maddison, D. (2011): Natural Resource and Environmental Economics, 4. Auflage, Pearson Education Limited, Harlow.

Philipp, M. (2002): Die Konstruktion des medialen Dopingdiskurses: Struktur und Strategie (1. Aufl .). Sozialwissenschaft. Wiesbaden: Dt. Univ.-Verl.

Pitsch, W. und Emrich E. (2012): The Frequency of Doping in Elite Sport - Results of a Replication Study, International Review for the Sociology of Sport, 47, 559580 .

Pitsch, W., Emrich, E. und Klein, M. (2005): Zur Häufigkeit des Dopings im Leistungssport. Ergebnisse eines www-surveys, Leipziger Sportwissenschaftliche Beiträge, XLVI, 63-77. 
Pitsch, W., Emrich, E. und Klein, M. (2007): Doping in Elite Sports in Germany, European Journal for Sport and Society 4, 2, 89-102.

Pitsch, W., Maats, P. und Emrich, E. (2009): Zur Häufigkeit des Dopings im deutschen Spitzensport, Magazin Forschung der Universität des Saarlandes, 1, 15-19.

Pitsch, W. (2009): The „Science of Doping“ Revisited: Fallacies of the Current Anti Doping Regime, European Journal of Sport Sciences, 9, 87-95.

Pitsch, W. (2011): Doping Controlls Between Test Theory and Ethics - Unintended Consequences of in Principle Imperfect Doping Tests. In: Emrich, E.; Pitsch, W. (eds.): Sport and Doping. The Analysis of an Antagonistic Symbiosis. Frankfurt Main: Lang, 101-113.

Popitz, H. (1968): Über die Präventivwirkung des Nichtwissens. Dunkelziffer, Norm, Strafe. Tübingen: Mohr.

Schetsche, M (2001): Metatheorie und Analyseprogramm - Zum Doppelstatus der relativistischen Problemtheorie. Soziale Probleme, Zeitschrift für soziale Probleme und soziale Kontrolle, 12, 28-44.

Schetsche, M. (1996). Die Karriere sozialer Probleme. Soziologische Einführung. München: Oldenbourg.

Schetsche, M. (2000). Wissenssoziologie sozialer Probleme. Grundlegung einer relativistischen Problemtheorie. Wiesbaden. Westdeutscher Verlag.

Spiller, C. (2012). Epo war gestern, Doping wird ansteckend - ZeitOnline 22.02.2012 (http://www.zeit.de/sport/2012-02/doping-ansteckend-gendoping-gefahr). 